\title{
Proteomic study reveals a functional network of cancer markers in the G1-Stage of the breast cancer cell cycle
}

Milagros J Tenga and Iulia M Lazar

\begin{abstract}
Background: Cancer cells are characterized by a deregulated cell cycle that facilitates abnormal proliferation by allowing cells to by-pass tightly regulated molecular checkpoints such as the G1/S restriction point. To facilitate early diagnosis and the identification of new drug targets, current research efforts focus on studies that could lead to the development of protein panels that collectively can improve the effectiveness of our response to the detection of a life-threatening disease.
\end{abstract}

Methods: Estrogen-responsive MCF-7 cells were cultured and arrested by serum deprivation in the G1-stage of the cell cycle, and fractionated into nuclear and cytoplasmic fractions. The protein extracts were trypsinized and analyzed by liquid chromatography - mass spectrometry (MS), and the data were interpreted with the Thermo Electron Bioworks software. Biological characterization of the data, selection of cancer markers, and identification of protein interaction networks was accomplished with a combination of bioinformatics tools provided by GoMiner, DAVID and STRING.

Results: The objective of this work was to explore via MS proteomic profiling technologies and bioinformatics data mining whether randomly identified cancer markers can be associated with the G1-stage of the cell cycle, i.e., the stage in which cancer cells differ most from normal cells, and whether any functional networks can be identified between these markers and placed in the broader context of cell regulatory pathways. The study enabled the identification of over 2000 proteins and 153 cancer markers, and revealed for the first time that the G1-stage of the cell cycle is not only a rich source of cancer markers, but also a host to an intricate network of functional relationships within the majority of these markers. Three major clusters of interacting proteins emerged:

(a) signaling, (b) DNA repair, and (c) oxidative phosphorylation.

Conclusions: The identification of cancer marker regulatory components that act not alone, but within networks, represents an invaluable resource for elucidating the moxlecular mechanisms that govern the uncontrolled proliferation of cancer cells, as well as for catalyzing the development of protein panels with biomarker and drug target potential, screening tests with improved sensitivity and specificity, and novel cancer therapies aimed at pursuing multiple drug targets.

Keywords: Cell cycle, Cancer markers, Proteomics, Mass spectrometry

\footnotetext{
* Correspondence: malazar@vt.edu

Department of Biological Sciences, Virginia Polytechnic Institute and State

University, 1981 Kraft Drive, Blacksburg, VA 24061, USA
} 


\section{Background}

Howard and Pelc described four consecutive phases of the cell cycle: G1, S, G2 and M [1]. Each phase needs to be completed before the next one can proceed: the G1phase is a period of growth in preparation for replication, the S-phase a period in which the DNA content is duplicated, the G2-phase a period of growth in preparation for mitosis, and the M-phase a period in which the cell divides into two identical daughter cells. Multiple regulatory events, termed checkpoints, verify whether certain cellular processes have occurred properly before allowing the cells to proceed from one phase to another [2-6]. For example, DNA damage checkpoints at the G1/ $\mathrm{S}$ and $\mathrm{G} 2 / \mathrm{M}$ transition boundaries, and spindle checkpoints during the M-phase, have been recognized. These checkpoints allow either for DNA repair, or correct chromosome alignment on the mitotic spindle, respectively, before the next steps of the cell cycle can proceed. In 1978, Pardee described the G1/S restriction point (R-point) as an essential regulatory event in the G1phase [3]. The period before the R-point is uniquely sensitive to growth factor stimulation, and in the absence of mitogenic signaling, normal cells exit the cell cycle and enter a reversible dormant/quiescent state termed G0. Alternatively, in the presence of major perturbations in the cell cycle regulatory machinery, such as DNA damage, normal cells attempt to repair such damage, or in the case of failure, commit apoptosis. Unlike normal cells, cancer cells evolved the ability to evade the restriction point and continue through the cell cycle even if DNA damage is detected. After the R-point, both normal and cancer cells are unaffected by the removal of growth factors or other deregulatory events, and enter the Sphase, committing to a round of cell division. Therefore, the R-point emerges as the most critical point in cell cycle control. Key to its regulation is the phosphorylation of the retinoblastoma protein (pRb or RB1) by active cyclin D-CDK4/6 and cyclin E-CDK2 complexes in early and late G1, respectively, an event that results in the release of E2F transcription factors that signal the cell to continue into the S-phase, replicate and proliferate. Hundreds of E2F target genes that are involved in DNA replication and cell cycle signaling, as well as DNA damage repair, programmed cell death, development and cell differentiation, have been identified. Traditional molecular biology and biochemistry approaches have greatly contributed to understanding breast cancer cell cycle regulation. However, the introduction of high-throughput genomic and proteomic methods, and the escalating development of novel bioinformatics tools, have revolutionized cancer research. Large lists of genes and proteins involved in important biological processes are generated with the aim of providing a comprehensive picture of all concurring events in a cell, the challenge continuing to rest with the interpretation of such voluminous data. As the mechanisms used by cancer cells to escape the Rrestriction point continue to remain unclear, the objective of this study was to use mass spectrometry technologies to generate a comprehensive map of proteins that are expressed in the critical G1-stage of the cell cycle in a representative model system of ER + breast cancer such as MCF-7, and make use of bioinformatics tools to explore: (a) whether cancer marker proteins reported by previous studies (rather unrelated) can be associated with this stage of the cell cycle, (b) whether a particular subcellular localization is characteristic for these proteins, and (c) whether these markers form regulatory networks that promote cell proliferation and can be placed in a broader context of cancer-relevant functional roles to advance a panel with biomarker and drug target potential.

\section{Methods}

\section{Cell processing}

MCF-7 cells (ATCC, Manassas, VA) were grown in EMEM with $10 \% \mathrm{FBS}$ and $10 \mu \mathrm{g} / \mathrm{mL}$ bovine insulin, in an incubator at $37^{\circ} \mathrm{C}$ with $5 \% \mathrm{CO}_{2}[7,8]$. The cells were arrested in the G1-phase by serum-deprivation for $48 \mathrm{~h}$, in a medium consisting of DMEM and $4 \mathrm{mM} \mathrm{L-glutam-}$ ine, harvested, separated into nuclear and cytoplasmic fractions (Cell Lytic ${ }^{\text {Tu }}$ NuCLEAR ${ }^{\text {mu }}$ extraction kit, Sigma, St. Louis, MO), digested with trypsin (Promega Corporation (Madison, WI) at $37^{\circ} \mathrm{C}$ for $24 \mathrm{~h}$ (50:1 substrate:enzyme ratio), and analyzed by nano-liquid chromatography (LC)MS/MS with a linear trap quadrupole (LTQ/Thermo Electron Corporation, San Jose, CA) mass spectrometer. FACS analysis was performed with a Beckman Coulter EPICS XL-MCL analyzer (Brea, CA, USA). The protein content was measured by the Bradford assay on a SmartSpec Plus spectrophotometer (Bio-Rad, Hercules, CA). The sample analyzed by MS contained $2 \mu \mathrm{g} / \mu \mathrm{L}$ MCF-7 proteins. LC separations were performed with an Agilent 1100 LC system (Palo Alto, CA) and in-house prepared nanoseparation columns $(100 \mu \mathrm{m}$ i.d. x $12 \mathrm{~cm})$ packed with $5 \mu \mathrm{m}$ Zorbax SB-C18 particles. Common reagents were purchased from Sigma, cell culture media from ATCC and Invitrogen (Carlsbad, CA), and HPLC-solvents from Fisher Scientific (Fair Lawn, NJ). Sample preparation and LC-MS/MS analysis protocols were described in detail in previous manuscripts $[7,8]$.

\section{Data processing}

A minimally redundant Homo sapiens protein database from SwissProt (2008/40,009 entries) and the Bioworks 3.3 software (Thermo Electron) were used for protein identifications. Conditions for peptide selection included: only fully tryptic fragments with maximum two missed cleavages, no posttranslational modifications, peptide and fragment ion tolerances set at $2 \mathrm{amu}$ and 
1 amu, respectively,\% fragment ion coverage $>30 \%$ (from any combination of theoretical b, $y$ and a ions), all peptides matched to unique proteins in the database, and Sequest Xcorr vs. charge state parameters set at 1.9, 2.2 and 3.8 for singly, doubly and triply charged peptides, respectively. At the protein level, the Bioworks p-score threshold was set at $\leq 0.001$. Proteins matched by one unique peptide were considered only when could be identified in at least two biological states or replicates. A few proteins matched by a single peptide count were allowed in the analysis, due to their relevance, but the associated SwissProt IDs should be treated in such cases with prudence due to the possibility of existing protein isoforms that share the same peptide. The peptide $\mathrm{p}$ values were for these cases $<0.001$. False discovery rates (FDR) were determined by searching the raw data against a forward-reversed protein sequence database. FDRs were $<3 \%$ and $<1 \%$ at the protein and peptide levels, respectively. Specific parameter settings for the use of bioinformatics tools were: GoMiner included all evidence codes; STRING parameters were set to high confidence, $\leq 10$ interactors, network depth 1 and all active prediction methods; the DAVID enrichment p-score threshold was 1.3 (shown as -log transformed value), with a Homo sapiens background and classification stringency set to medium.

\section{Results and discussion}

\section{Sample and data analysis}

While the key events of the cell cycle control take place in the nucleus, a number of relevant signaling pathways activated by mitogenic stimuli proceed through the cytoplasm, prior to impacting the nuclear sequence of events. Furthermore, many proteins are shuttled between the cytoplasm and nucleus as a means of functional activation/ deactivation. To increase the number of identifiable proteins and generate a comprehensive map of the biological processes that unfold in the G1-stage of the cell cycle, the MCF-7 cells were separated into nuclear and cytoplasmic fractions. Three biological replicates were prepared to enable a confident selection of identifiable proteins, and five LC-MS/MS technical replicates were performed to maximize the number of identifiable proteins and the number of spectral counts per protein $[7,8]$. A total of six samples were generated from the two cell states [i.e., G1phase nuclear (G1N1, G1N2 and G1N3) and G1-phase cytoplasmic (G1C1, G1C2 and G1C3)] and a total of 30 LC-MS/MS analyses were performed. Reproducibility was assessed at every step of the analysis. The cell cycle distribution in each cell culture was evaluated by flow cytometry (Figure 1). The arrested cells were found primarily in G1 $(\sim 81 \%)$, and only a small proportion in S $(\sim 10 \%)$ and G2 $(\sim 7 \%)$, respectively. A bar graph illustrating the trend in protein identifications is provided in Figure 2. Each biological replicate displays cumulative protein identifications in $5 \mathrm{LC}-\mathrm{MS} / \mathrm{MS}$ analyses, and new protein identifications relative to the previous replicates. After MS data processing and filtering, in-house developed Perl-scripts were used for aligning protein and peptide spectral count data [9]. A total of 2375 proteins were identified, of which, 2000 with two or more spectral counts. The average number of identified proteins and matching counts in each of the 6 cell states was $1176(\mathrm{CV}=7.5 \%)$ and $4030(\mathrm{CV}=8.9 \%)$, respectively, with a total of 1515 proteins in the combined nuclear fractions and 1572 in the combined cytoplasmic fractions. The correlation coefficient of protein identifications based on spectral count data in any two biological replicates of a cell state reached values as high as $\mathrm{R}=0.96$, as shown in a representative comparison involving the G1N1 and G1N2 fractions containing a total of 1239
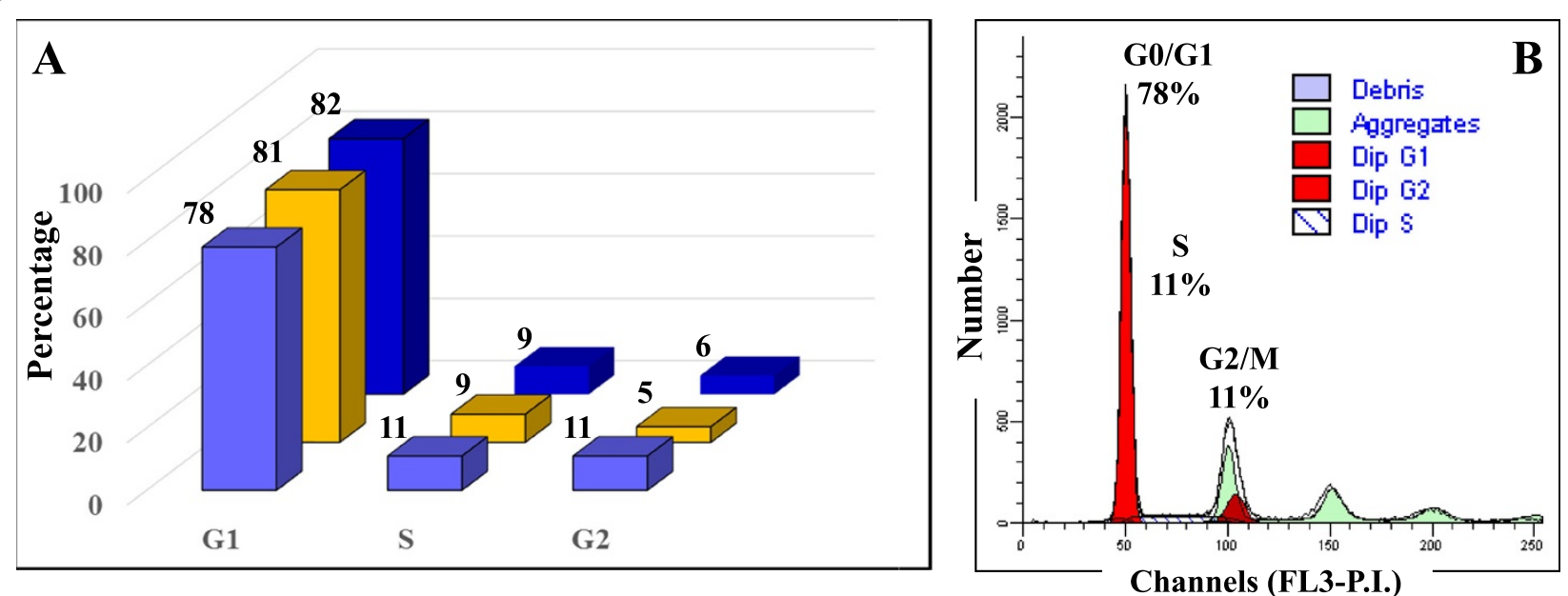

Figure 1 FACS analysis of MCF-7 cells. (A) Bar graph illustrating the reproducibility of cell cycle arrest in G1 by serum deprivation for 48 hours in three biological replicate cultures; (B) FACS diagram of G1-stage MCF-7 cells. 


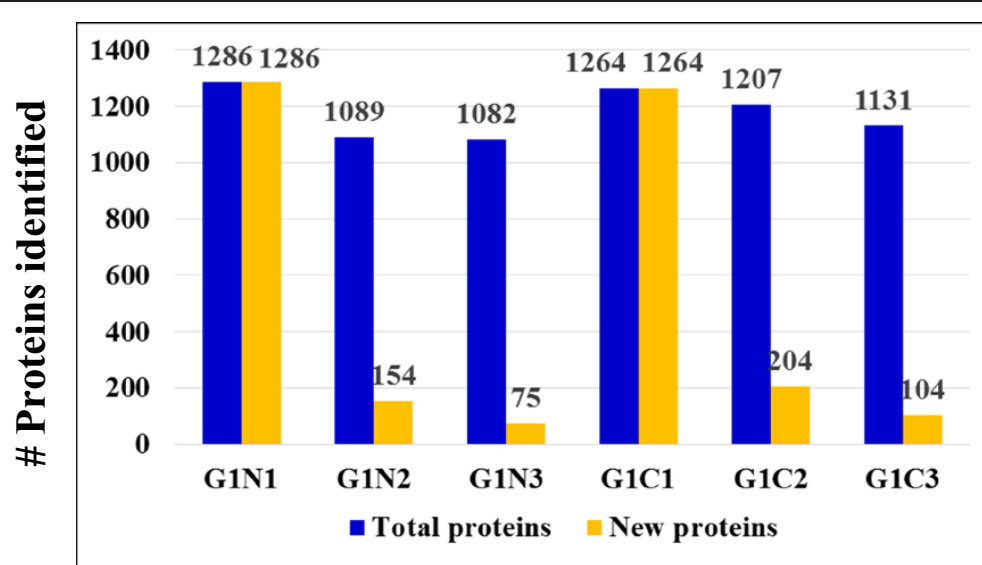

Biological states and replicates

Figure 2 Reproducibility of protein identifications in three biological replicates of G1N/G1C MCF-7 cells.

proteins (Figure 3A). As expected, however, due to biological and technical variability, the effective overlap of protein IDs between all three replicates did not exceed 75\% (Figure 3B). Nevertheless, the above described workflow enabled the identification of a sufficient number of proteins for extracting meaningful biological information despite the lack of a high-end mass spectrometer platform for performing the experiments. The ExPASy Proteomics Server [10], GoMiner [11], DAVID Bioinformatics Resources [12,13], STRING functional protein association networks 8.3 [14] and the GeneCards [15] bioinformatics tools were used for the functional interpretation of the data. GoMiner analysis revealed that the nuclear cell fractions comprised 57-62\% and $59-64 \%$ proteins with nuclear and cytoplasmic categorization, respectively. The cytoplasmic fractions comprised primarily cytoplasmic proteins (83-84\%), and only a small fraction of nuclear proteins (32-33\%). While complete separation of the two cell fractions was not experimentally achievable, the nuclear enrichment process resulted in an increase of the nuclear proteins from 15-20\% in a whole cell extract, to $>50 \%$ in the nuclear-enriched fraction. DAVID functional clustering of the MCF-7 proteins with two or more spectral counts returned over 150 clusters with enrichment scores $>1.3$. The large number of clusters reflects that the dataset is representative of a broad range of basic biological processes that occur in a cell. The top scoring clusters included processes related to the biosynthesis and processing of nucleotides, RNA, proteins and ATP, and to transport, proteasome and metabolism. Additional file 1 lists the identified proteins, their total spectral count, and their identification in the nuclear or cytoplasmic fractions. The overlapping nuclear/cytoplasmic categories (i.e., $30 \%$ ) included proteins with roles in gene expression/translation/protein biosynthesis, glycolysis, glucose/carbohydrate metabolism, and intracellular transport.

\section{Query for putative cancer markers}

Overall, from the list of 2375 proteins, GoMiner/DAVID categorization returned a considerable number of proteins involved in biological processes representative of all hallmarks of cancer $[6,16]$ that matched multiple pathways in the Kegg cancer diagram, i.e., proliferation,

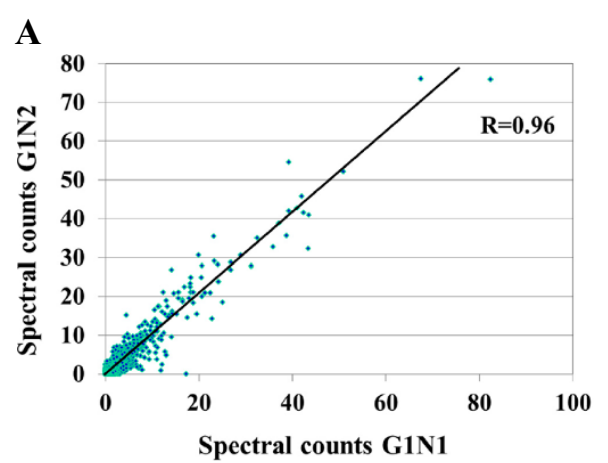

B

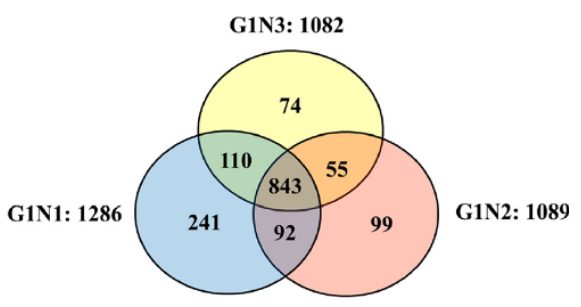

G1N1 + G1N2 + G1N3 = 1515 unique proteins

Figure 3 Protein overlaps among biological replicates of MCF-7 cells. (A) Scatter plot of protein identifications in two biological replicates of MCF-7 G1N cells (G1N1 vs. G1N2, total 1239 proteins). (B) Venn diagram of protein overlaps between three biological replicates of MCF-7 G1N cells. 
cell cycle, apoptosis, evasion of apoptosis, failed repair of genes, insensitivity to growth factors, sustained angiogenesis, and PPAR signaling [17]. The list of 2375 was queried for the presence of proteins with role in cancer development or with previously reported biomarker potential. The search in the DAVID disease database returned a list of 96 proteins associated with cancer, of which 51 proteins were matched to breast cancer. Table 1 enlists the identified markers and spectral count data, categorized according to biological processes of relevance to cancer. Additional file 2 enlists supplemental information such as associated $\mathrm{GO}$ biological processes, GO cellular compartments, GO molecular functions, Kegg pathways and associated diseases, STRING descriptions, full Sequest report and gene abbreviations. Table 1 was complemented with a set of 57 G1-stage proteins with known significance to cell cycle regulation and cancer $[6,12,15]$, shown as entries in italic, to amount to a total of 153 protein I.D.s.

Proteins of relevance to cancer, but not identified in the dataset, were included in the discussion, but were not included in the generation of lists, figures or the STRING diagrams. Cancer-relevant proteins were present in both nuclear (100 proteins) and cytoplasmic (102 proteins) fractions. Most importantly, a STRING protein-protein interaction diagram revealed a widespread connectivity between these randomly mapped cancer proteins, and redundant identification of the same categories with relevance to cell cycle regulation and proliferation, suggesting the possibility of a useful biomarker panel for diagnostic purposes, or of novel drug candidates that could be targeted synergistically in cancer therapy (Figure 4). Three main networks emerged from the list: (1) signaling and cell cycle regulation, (2) maintenance of genome integrity and DNA repair, and (3) oxidative phosphorylation, stress, energy production and metabolism. While the advocated protein panel is not necessarily specific to MCF-7, and while differential expression profiling was not the purpose of the present study, preliminary comparisons to non-tumorigenic G1arrested MCF-10 cells confirmed that roughly two thirds of the MCF-7 markers changed spectral counts more than 2-fold, and some even more than 10-fold, when comparted to MCF-10. The results also confirm that proteomic analysis of relevant cancerous cell states can capture in a single experiment protein panels that previously could be identified only by multiple studies, with various model systems, and using various biochemical/ biological approaches and tools. A subset of proteins displayed either very small, or, essentially, no change in spectral counts (APEX1, KU70/KU86, LEG3, PARP1, PGK1, PHB, PRDX2, PRKDC, RAC1, RHOA/RHOC, SHC1, TBB3/TBB5, TYB4, UCRI, ZO). Future work will discuss in detail the quantitative comparison of the two cell lines in both nuclear and cytoplasmic fractions. The functional relevance of the most prominent protein clusters that were identified within the three major categories, as well as their broader impact on cancer cell proliferation is discussed below.

\section{Cell cycle regulation, proliferation and checkpoint}

Among the key cell cycle and proliferation regulators, the cell cycle and mitotic checkpoint proteins with essential roles in maintaining the integrity of the cell division process (PRKDC, TP53BP1, BUB3, RB1), the proliferation markers (PCNA, KI-67, 14-3-3 sigma, $\mathrm{PHB})$, the cyclin dependent kinases CDK2 and CDK1 (CDC2), the alpha and beta catalytic subunits of the protein phosphatase type $1 \mathrm{PP} 1$ (PP1A and PP1B), and a series of other proteins that control transcription regulation, chromatin maintenance, mitosis, signaling and proteasome degradation, were identified. The phosphorylation of the RB1 protein by cyclin D1-CDK4/6 complexes plays an important role in cell advancement through the cell cycle and the regulation of the R-point: the unphosphorylated form is present in G0, hypophosphorylation correlates to entry into $\mathrm{G} 1$, and hyperphosphorylation is concurrent with passing of the restriction point and completion of the cell cycle. Upon exit from mitosis, the phosphate groups are removed by the Ser/Thr-protein phosphatase PP1 proteins [6]. Along with the cyclin-CDK complexes, protein phosphatases play an important role in cell cycle control through their modulation of signal transduction pathways. Active CDK2 is essential after the Rpoint, in late $\mathrm{G} 1$ and $S$, one of its roles in $S$ being the phosphorylation of pol- $\alpha$ :primase which promotes DNA synthesis in S. Active CDK1 is essential in the M-phase, and also for entry into the S-phase in the absence of CDK2 [18]. Along with RB1, the guardian of the R-point gate, BUB3 acts as an M-phase mitotic spindle assembly checkpoint protein and inhibitor of the anaphase promoting complex (APC) that tags cell cycle proteins with ubiquitin for proteasomal degradation by the $26 \mathrm{~S}$ proteasome [15]. TP53BP, through its association with p53, plays a key role in DNA damage response and transcription regulation, and PRKDC, a Ser/Thr kinase, in association with XRCC5/6 is a first-line responder and sensor of DNA damage. In parallel with their essential function in DNA repair, these proteins have additional roles in cell cycle regulation [12-15].

Consistent with cancer cell propagation, a number of known proliferation markers were detectable, i.e., PCNA, antigen Ki-67, 14-3-3 sigma and prohibitin-PHB [15,19]. PCNA is involved in the control of eukaryotic DNA replication, and displays high expression levels in proliferating cells. Ki-67 is a marker of proliferation, being detectable in all stages of the cell cycle, except G0. 14-3-3 sigma is an adaptor protein which is involved in multiple signaling 
Table 1 Biological categorization of MCF-7 proteins matched in the DAVID disease/cancer database (entries in italic are not all cancer markers, but were included in the list due to their functional relevance to the marker proteins)

\begin{tabular}{|c|c|c|c|c|c|}
\hline ID & Protein name & MW & Total counts & Cancer & Breast cance \\
\hline \multicolumn{6}{|c|}{ Cell cycle/division/check-point/proliferation } \\
\hline O43684 & BUB3_HUMAN Mitotic checkpoint protein BUB3 & 37131.2 & 78 & Cancer & Breast cancer \\
\hline P06400 & RB_HUMAN Retinoblastoma-associated protein & 106091.7 & 15 & Cancer & Breast cancer \\
\hline P12004 & PCNA_HUMAN Proliferating cell nuclear antigen & 28750.3 & 17 & Cancer & \\
\hline P35232 & PHB_HUMAN Prohibitin & 29785.9 & 96 & Cancer & Breast cancer \\
\hline P78527 & PRKDC_HUMAN DNA-dependent protein kinase catalytic subunit & 468786.9 & 889 & Cancer & Breast cancer \\
\hline P07437 & TBB5_HUMAN Tubulin beta chain & 49639 & 230 & Cancer & Breast cancer \\
\hline Q13509 & TBB3_HUMAN Tubulin beta-3 chain & 50400.3 & 127 & Cancer & Breast cancer \\
\hline Q14980 & NUMA1_HUMAN Nuclear mitotic apparatus protein 1 & 238113.2 & 505 & Cancer & Breast cancer \\
\hline Q12888 & TP53B_HUMAN Tumor suppressor p53-binding protein 1 & 213440.8 & 7 & Cancer & Breast cancer \\
\hline Q6P1J9 & CDC73_HUMAN Parafibromin & 60539.2 & 12 & Cancer & \\
\hline P37231 & PPARG_HUMAN Peroxisome proliferator-activated receptor gamma & 57583.2 & 5 & Cancer & Breast cancer \\
\hline P46013 & KI67_HUMAN Antigen KI-67 & 358471.9 & 50 & & \\
\hline P31947 & 1433S_HUMAN 14-3-3 protein sigma & 27756.7 & 67 & & \\
\hline Q81X12 & CCAR__HUMAN Cell division cycle and apoptosis regulator protein 1 & 132738.6 & 72 & & \\
\hline P06493 & CDC2_HUMAN Cell division control protein 2 homolog & 34073.9 & 20 & & \\
\hline P62136 & PP1A_HUMAN Serine/threonine-protein phosphatase PP1-alpha catalytic subunit & 37487.8 & 179 & & \\
\hline P62140 & PP1B_HUMAN Serine/threonine-protein phosphatase PP1-beta catalytic subunit & 37162.6 & 9 & & \\
\hline Q13616 & CUL1_HUMAN Cullin-1 & 89622 & 12 & & \\
\hline Q13547 & HDAC1_HUMAN Histone deacetylase 1 & 55067.8 & 120 & & \\
\hline Q92769 & HDAC2_HUMAN Histone deacetylase 2 & 55328.8 & 31 & & \\
\hline \multicolumn{6}{|c|}{ Apoptosis } \\
\hline 014737 & PDCD5_HUMAN Programmed cell death protein 5 & 14276.3 & 6 & Cancer & \\
\hline Q07812 & BAX_HUMAN Apoptosis regulator BAX & 21170.8 & 1 & Cancer & \\
\hline Q13153 & PAK1_HUMAN Serine/threonine-protein kinase PAK 1 & 60608.8 & 4 & Cancer & \\
\hline O43464 & HTRA2_HUMAN Serine protease HTRA2, mitochondrial precursor & 48811 & 5 & & \\
\hline O75340 & PDCD6_HUMAN Programmed cell death protein 6 & 21854.8 & 103 & & \\
\hline O95831 & AIFM1_HUMAN Apoptosis-inducing factor 1, mitochondrial precursor & 66859 & 57 & & \\
\hline P55957 & BID_HUMAN BH3-interacting domain death agonist & 21981.1 & 7 & & \\
\hline Q13158 & FADD_HUMAN Protein FADD & 23264.9 & 10 & & \\
\hline Q81X12 & CCAR1_HUMAN Cell division cycle and apoptosis regulator protein 1 & 132738.6 & 72 & & \\
\hline Q8N8D1 & PDCD7_HUMAN Programmed cell death protein 7 & 54666.3 & 4 & & \\
\hline Q96IZ0 & PAWR_HUMAN PRKC apoptosis WT1 regulator protein & 36545.5 & 5 & & \\
\hline Q9BTC0 & DIDO1_HUMAN Death-inducer obliterator 1 & 243720.8 & 76 & & \\
\hline Q9BZZ5 & API5_HUMAN Apoptosis inhibitor 5 & 57525.2 & 75 & & \\
\hline Q9NR28 & DBLOH_HUMAN Diablo homolog, mitochondrial precursor & 27113.7 & 8 & & \\
\hline Q9NYF8 & BCLF1_HUMAN BCl-2-associated transcription factor 1 & 106058.7 & 80 & & \\
\hline Q9ULZ3 & ASC_HUMAN Apoptosis-associated speck-like protein containing a CARD & 21613.3 & 43 & & \\
\hline \multicolumn{6}{|c|}{ DNA Repair } \\
\hline 000255 & MEN1_HUMAN Menin & 67951 & 4 & Cancer & \\
\hline P09874 & PARP1_HUMAN Poly [ADP-ribose] polymerase 1 & 113012.4 & 342 & Cancer & Breast cancer \\
\hline P12004 & PCNA_HUMAN Proliferating cell nuclear antigen & 28750.3 & 17 & Cancer & \\
\hline P12956 & KU70_HUMAN ATP-dependent DNA helicase 2 subunit 1 & 69799.2 & 398 & Cancer & Breast cancer \\
\hline
\end{tabular}


Table 1 Biological categorization of MCF-7 proteins matched in the DAVID disease/cancer database (entries in italic are not all cancer markers, but were included in the list due to their functional relevance to the marker proteins) (Continued)

\begin{tabular}{|c|c|c|c|c|c|}
\hline P13010 & KU86_HUMAN ATP-dependent DNA helicase 2 subunit 2 & 82652.4 & 367 & Cancer & Breast cancer \\
\hline P16455 & MGMT_HUMAN Methylated-DNA--protein-cysteine methyltransferase & 21632.2 & 22 & Cancer & Breast cancer \\
\hline P18074 & ERCC2_HUMAN TFIIH basal transcription factor complex helicase subunit & 86854.3 & 3 & Cancer & Breast cancer \\
\hline P18887 & XRCC1_HUMAN DNA-repair protein XRCC1 & 69483.1 & 15 & Cancer & Breast cancer \\
\hline P20585 & MSH3_HUMAN DNA mismatch repair protein Msh3 & 127376.1 & 5 & Cancer & \\
\hline P27695 & APEX1_HUMAN DNA-(apurinic or apyrimidinic site) lyase & 35532.2 & 78 & Cancer & Breast cancer \\
\hline P29372 & 3MG_HUMAN DNA-3-methyladenine glycosylase & 32842.8 & 36 & Cancer & \\
\hline P35244 & RFA3_HUMAN Replication protein A 14 kDa subunit & 13559.9 & 29 & Cancer & \\
\hline P43246 & MSH2_HUMAN DNA mismatch repair protein Msh2 & 104676.8 & 21 & Cancer & \\
\hline P46063 & RECQ1_HUMAN ATP-dependent DNA helicase Q1 & 73409.1 & 10 & Cancer & \\
\hline P49916 & DNL3_HUMAN DNA ligase 3 & 102625.5 & 4 & Cancer & Breast cancer \\
\hline P52701 & MSH6_HUMAN DNA mismatch repair protein MSH6 & 152688.4 & 37 & Cancer & \\
\hline P54727 & RD23B_HUMAN UV excision repair protein RAD23 homolog B & 43144.6 & 78 & Cancer & Breast cancer \\
\hline P78527 & PRKDC_HUMAN DNA-dependent protein kinase catalytic subunit & 468786.9 & 889 & Cancer & Breast cancer \\
\hline Q01831 & XPC_HUMAN DNA-repair protein complementing XP-C cells & 105915.3 & 14 & Cancer & Breast cancer \\
\hline Q12888 & TP53B_HUMAN Tumor suppressor p53-binding protein 1 & 213440.8 & 7 & Cancer & Breast cancer \\
\hline Q92466 & DDB2_HUMAN DNA damage-binding protein 2 & 47833.4 & 5 & Cancer & \\
\hline Q92878 & RAD50_HUMAN DNA repair protein RAD50 & 153795.8 & 11 & Cancer & Breast cancer \\
\hline Q9UBB5 & MBD2_HUMAN Methyl-CpG-binding domain protein 2 & 43228 & 7 & Cancer & Breast cancer \\
\hline Q9UHN1 & DPOG2_HUMAN DNA polymerase subunit gamma-2, mitochondrial precursor & 54876.3 & 7 & Cancer & \\
\hline P39748 & FEN1_HUMAN Flap endonuclease 1 & 42566.1 & 114 & & \\
\hline \multicolumn{6}{|c|}{ Angiogenesis } \\
\hline P13489 & RINI_HUMAN Ribonuclease inhibitor & 49941.2 & 17 & Cancer & \\
\hline P40763 & STAT3_HUMAN Signal transducer and activator of transcription 3 & 88011.4 & 13 & Cancer & \\
\hline Q16539 & MK14_HUMAN Mitogen-activated protein kinase 14 & 41267.1 & 2 & & \\
\hline Q14119 & VEZF1_HUMAN Vascular endothelial zinc finger 1 & 56326.5 & 9 & & \\
\hline Q13685 & AAMP_HUMAN Angio-associated migratory cell protein & 46721.5 & 1 & & \\
\hline Q9UQB8 & BAIP2_HUMAN Brain-specific angiogenesis inhibitor 1-associated protein & 60829.7 & 5 & & \\
\hline Q92793 & CBP_HUMAN CREB-binding protein & 265180.1 & 2 & & \\
\hline \multicolumn{6}{|c|}{ Migration/invasion/adhesion/metastasis } \\
\hline P07858 & CATB_HUMAN Cathepsin B precursor & 37796.8 & 6 & Cancer & \\
\hline P15941 & MUC1_HUMAN Mucin-1 precursor & 121999.6 & 4 & Cancer & \\
\hline P17931 & LEG3_HUMAN Galectin-3 & 26172.1 & 77 & Cancer & \\
\hline P35222 & CTNB1_HUMAN Catenin beta-1 & 85442.3 & 1 & Cancer & \\
\hline P62328 & TYB4_HUMAN Thymosin beta-4 & 5049.5 & 15 & Cancer & \\
\hline Q06124 & PTN11_HUMAN Tyrosine-protein phosphatase non-receptor type 11 & 68393.4 & 2 & Cancer & \\
\hline Q08380 & LG3BP_HUMAN Galectin-3-binding protein precursor & 65289.4 & 2 & Cancer & \\
\hline Q13153 & PAK1_HUMAN Serine/threonine-protein kinase PAK 1 & 60608.8 & 4 & Cancer & \\
\hline Q13330 & MTA1_HUMAN Metastasis-associated protein MTA1 & 80737.4 & 13 & Cancer & Breast cancer \\
\hline O60716 & CTND1_HUMAN Catenin delta-1 & 108103.3 & 37 & & \\
\hline P07339 & CATD_HUMAN Cathepsin D precursor & 44523.7 & 105 & & \\
\hline P07355 & ANXA2_HUMAN Annexin A2 & 38579.8 & 627 & & \\
\hline
\end{tabular}


Table 1 Biological categorization of MCF-7 proteins matched in the DAVID disease/cancer database (entries in italic are not all cancer markers, but were included in the list due to their functional relevance to the marker proteins) (Continued)

\begin{tabular}{|c|c|c|c|c|c|}
\hline P09493 & TPM1_HUMAN Tropomyosin alpha-1 chain & 32688.7 & 91 & & \\
\hline P12814 & ACTN1_HUMAN Alpha-actinin-1 & 102992.7 & 441 & & \\
\hline P23528 & COF1_HUMAN Cofilin-1 & 18490.7 & 508 & & \\
\hline P35221 & CTNA1_HUMAN Catenin alpha-1 & 100008.6 & 100 & & \\
\hline P35580 & MYH10_HUMAN Myosin-10 & 228796.8 & 136 & & \\
\hline P60953 & CDC42_HUMAN Cell division control protein 42 homolog precursor & 21296.9 & 3 & & \\
\hline P61586 & RHOA_HUMAN Transforming protein RhoA precursor & 21754.1 & 12 & & \\
\hline P62258 & 1433E_HUMAN 14-3-3 protein epsilon & 29155.4 & 136 & & \\
\hline P63000 & RAC1_HUMAN Ras-related C3 botulinum toxin substrate 1 precursor & 21436.3 & 9 & & \\
\hline Q07157 & ZO1_HUMAN Tight junction protein ZO-1 & 195338.8 & 32 & & \\
\hline Q13418 & ILK_HUMAN Integrin-linked protein kinase & 51386 & 43 & & \\
\hline Q9UDY2 & ZO2_HUMAN Tight junction protein ZO-2 & 133890.2 & 5 & & \\
\hline Q13685 & AAMP_HUMAN Angio-associated migratory cell protein & 46721.5 & 1 & & \\
\hline P08134 & RHOC_HUMAN Rho-related GTP-binding protein RhoC precursor & 21992.3 & 4 & & \\
\hline \multicolumn{6}{|c|}{ Differentiation } \\
\hline P15531 & NDKA_HUMAN Nucleoside diphosphate kinase A & 17137.7 & 154 & Cancer & Breast cancer \\
\hline P33076 & C2TA_HUMAN MHC class II transactivator & 123379.3 & 2 & Cancer & \\
\hline O60869 & EDF1_HUMAN Endothelial differentiation-related factor 1 & 16358.9 & 9 & & \\
\hline O95758 & RODI_HUMAN Regulator of differentiation 1 & 56466.5 & 15 & & \\
\hline P09382 & LEG1_HUMAN Galectin-1 & 14706.2 & 7 & & \\
\hline P17931 & LEG3_HUMAN Galectin-3 & 26172.1 & 77 & Cancer & \\
\hline P23771 & GATA3_HUMAN Trans-acting T-cell-specific transcription factor GATA3 & 47885.3 & 3 & & \\
\hline P35221 & CTNA1_HUMAN Catenin alpha-1 & 100008.6 & 100 & & \\
\hline P37231 & PPARG_HUMAN Peroxisome proliferator-activated receptor gamma & 57583.2 & 5 & Cancer & Breast cancer \\
\hline P40763 & STAT3_HUMAN Signal transducer and activator of transcription 3 & 88011.4 & 13 & Cancer & \\
\hline Q8TB36 & GDAP1_HUMAN Ganglioside-induced differentiation-associated protein 1 & 41225.8 & 2 & & \\
\hline \multicolumn{6}{|c|}{ Signaling } \\
\hline 000170 & AIP_HUMAN AH receptor-interacting protein & 37640.2 & 18 & Cancer & Breast cancer \\
\hline O14908 & GIPC1_HUMAN PDZ domain-containing protein GIPC1 & 36026.7 & 34 & Cancer & Breast cancer \\
\hline P01112 & RASH_HUMAN GTPase HRas precursor & 21284.6 & 6 & Cancer & Breast cancer \\
\hline P02786 & TFR1_HUMAN Transferrin receptor protein 1 & 84818 & 19 & Cancer & Breast cancer \\
\hline P08107 & HSP71_HUMAN Heat shock 70 kDa protein 1 & 70009.2 & 560 & Cancer & \\
\hline P11142 & HSP7C_HUMAN Heat shock cognate 71 kDa protein & 70854.4 & 895 & Cancer & \\
\hline P15941 & MUC1_HUMAN Mucin-1 precursor & 121999.6 & 4 & Cancer & \\
\hline P16615 & AT2A2_HUMAN Sarcoplasmic/endoplasmic reticulum calcium ATPase 2 & 114682.7 & 8 & Cancer & \\
\hline P29353 & SHC1_HUMAN SHC-transforming protein 1 & 62812.6 & 8 & Cancer & Breast cancer \\
\hline P33076 & C2TA_HUMAN MHC class II transactivator & 123379.3 & 2 & Cancer & \\
\hline P34931 & HS71L_HUMAN Heat shock $70 \mathrm{kDa}$ protein $1 \mathrm{~L}$ & 70331.5 & 482 & Cancer & \\
\hline P35222 & CTNB1_HUMAN Catenin beta-1 & 85442.3 & 1 & Cancer & \\
\hline P37231 & PPARG_HUMAN Peroxisome proliferator-activated receptor gamma & 57583.2 & 5 & Cancer & Breast cancer \\
\hline P40763 & STAT3_HUMAN Signal transducer and activator of transcription 3 & 88011.4 & 13 & Cancer & \\
\hline P78527 & PRKDC_HUMAN DNA-dependent protein kinase catalytic subunit & 468786.9 & 889 & Cancer & Breast cancer \\
\hline
\end{tabular}


Table 1 Biological categorization of MCF-7 proteins matched in the DAVID disease/cancer database (entries in italic are not all cancer markers, but were included in the list due to their functional relevance to the marker proteins) (Continued)

\begin{tabular}{|c|c|c|c|c|c|}
\hline Q04206 & TF65_HUMAN Transcription factor p65 & 60181.6 & 2 & Cancer & \\
\hline Q06124 & PTN11_HUMAN Tyrosine-protein phosphatase non-receptor type 11 & 68393.4 & 2 & Cancer & \\
\hline Q07812 & BAX_HUMAN Apoptosis regulator BAX & 21170.8 & 1 & Cancer & \\
\hline Q13085 & COA1_HUMAN Acetyl-CoA carboxylase 1 & 265382.7 & 1 & Cancer & Breast cancer \\
\hline Q13153 & PAK1_HUMAN Serine/threonine-protein kinase PAK 1 & 60608.8 & 4 & Cancer & \\
\hline Q14653 & IRF3_HUMAN Interferon regulatory factor 3 & 47189.7 & 2 & Cancer & \\
\hline Q15796 & SMAD2_HUMAN Mothers against decapentaplegic homolog 2 & 52272.8 & 1 & Cancer & \\
\hline Q5JWF2 & GNAS1_HUMAN Guanine nucleotide-binding protein G(s) subunit alpha isoforms XLas & 110955.6 & 67 & Cancer & Breast cancer \\
\hline Q7LG56 & RIR2B_HUMAN Ribonucleoside-diphosphate reductase subunit M2 B & 40710.5 & 5 & Cancer & \\
\hline Q8WUF5 & IASPP_HUMAN RelA-associated inhibitor & 89036 & 15 & Cancer & \\
\hline Q92466 & DDB2_HUMAN DNA damage-binding protein 2 & 47833.4 & 5 & Cancer & \\
\hline Q96RG5 & Q96RG5_HUMAN Insulin receptor substrate 2 insertion mutant & 137347.9 & 1 & Cancer & Breast cancer \\
\hline P31947 & 1433S_HUMAN 14-3-3 protein sigma & 27756.7 & 67 & & \\
\hline P62993 & GRB2_HUMAN Growth factor receptor-bound protein 2 & 25190.4 & 32 & & \\
\hline P46108 & CRK_HUMAN Proto-oncogene C-crk & 33810 & 18 & & \\
\hline Q16539 & MK14_HUMAN Mitogen-activated protein kinase 14 & 41267.1 & 2 & & \\
\hline P42224 & STAT1_HUMAN Signal transducer and activator of transcription 1-alpha/be & 87279.6 & 62 & & \\
\hline Q13547 & HDAC1_HUMAN Histone deacetylase 1 & 55067.8 & 120 & & \\
\hline Q92769 & HDAC2_HUMAN Histone deacetylase 2 & 55328.8 & 31 & & \\
\hline P56545 & CTBP2_HUMAN C-terminal-binding protein 2 & 48914.2 & 26 & & \\
\hline Q13363 & CTBP1_HUMAN C-terminal-binding protein 1 & 47505.6 & 8 & & \\
\hline Q92793 & CBP_HUMAN CREB-binding protein & 265180.1 & 2 & & \\
\hline Q13616 & CUL1_HUMAN Cullin-1 & 89622 & 12 & & \\
\hline P62136 & PP1A_HUMAN Serine/threonine-protein phosphatase PP1-alpha catalytic subunit & 37487.8 & 179 & & \\
\hline P62140 & PPIB_HUMAN Serine/threonine-protein phosphatase PP1-beta catalytic subunit & 37162.6 & 9 & & \\
\hline P06493 & CDC2_HUMAN Cell division control protein 2 homolog & 34073.9 & 20 & & \\
\hline P61586 & RHOA_HUMAN Transforming protein RhoA precursor & 21754.1 & 12 & & \\
\hline P08134 & RHOC_HUMAN Rho-related GTP-binding protein RhoC precursor & 21992.3 & 4 & & \\
\hline \multicolumn{6}{|c|}{ Oxidative processes/redox } \\
\hline P00441 & SODC_HUMAN Superoxide dismutase [Cu-Zn] & 15925.9 & 35 & Cancer & Breast cancer \\
\hline P03891 & NU2M_HUMAN NADH-ubiquinone oxidoreductase chain 2 & 38934.8 & 4 & Cancer & Breast cancer \\
\hline P04040 & CATA_HUMAN Catalase & 59718.9 & 3 & Cancer & Breast cancer \\
\hline P04179 & SODM_HUMAN Superoxide dismutase [Mn], mitochondrial precursor & 24706.6 & 1 & Cancer & Breast cancer \\
\hline P10599 & THIO_HUMAN Thioredoxin & 11729.7 & 28 & Cancer & Breast cancer \\
\hline P15559 & NQO1_HUMAN NAD(P)H dehydrogenase [quinone] 1 & 30848 & 40 & Cancer & Breast cancer \\
\hline P16435 & NCPR_HUMAN NADPH-cytochrome P450 reductase & 76641.4 & 22 & Cancer & Breast cancer \\
\hline P21912 & DHSB_HUMAN Succinate dehydrogenase [ubiquinone] iron-sulfur subunit & 31608.9 & 5 & Cancer & \\
\hline P47985 & UCRI_HUMAN Cytochrome b-c1 complex subunit Rieske, mitochondrial precursor & 29649.4 & 2 & Cancer & Breast cancer \\
\hline Q99757 & THIOM_HUMAN Thioredoxin, mitochondrial precursor & 18371.6 & 1 & Cancer & Breast cancer \\
\hline Q07973 & CP24A_HUMAN Cytochrome P450 24A1, mitochondrial precursor & 58837.6 & 15 & Cancer & Breast cancer \\
\hline P30041 & PRDX6_HUMAN Peroxiredoxin-6 & 25019.2 & 150 & & \\
\hline P30044 & PRDX5_HUMAN Peroxiredoxin-5, mitochondrial precursor & 22012.5 & 110 & & \\
\hline
\end{tabular}


Table 1 Biological categorization of MCF-7 proteins matched in the DAVID disease/cancer database (entries in italic are not all cancer markers, but were included in the list due to their functional relevance to the marker proteins) (Continued)

\begin{tabular}{|c|c|c|c|c|c|}
\hline P30048 & PRDX3_HUMAN Thioredoxin-dependent peroxide reductase, mitochondrial precursor & 27675.2 & 48 & & \\
\hline P32119 & PRDX2_HUMAN Peroxiredoxin-2 & 21878.2 & 102 & & \\
\hline Q06830 & PRDX1_HUMAN Peroxiredoxin-1 & 22096.3 & 191 & & \\
\hline \multicolumn{6}{|c|}{ Various metabolic functions and DNA/RNA processing } \\
\hline 043708 & MAAI_HUMAN Maleylacetoacetate isomerase & 24166.7 & 4 & Cancer & Breast cancer \\
\hline P00390 & GSHR_HUMAN Glutathione reductase, mitochondrial precursor & 56221 & 1 & Cancer & Breast cancer \\
\hline P00492 & HPRT_HUMAN Hypoxanthine-guanine phosphoribosyltransferase & 24563.6 & 32 & Cancer & \\
\hline P00558 & PGK1_HUMAN Phosphoglycerate kinase 1 & 44586.2 & 466 & Cancer & \\
\hline P07099 & HYEP_HUMAN Epoxide hydrolase 1 & 52915 & 9 & Cancer & Breast cancer \\
\hline P11172 & PYR5_HUMAN Uridine 5'-monophosphate synthase & 52188.7 & 11 & Cancer & \\
\hline P11586 & C1TC_HUMAN C-1-tetrahydrofolate synthase, cytoplasmic & 101495.6 & 149 & Cancer & Breast cancer \\
\hline P15531 & NDKA_HUMAN Nucleoside diphosphate kinase A & 17137.7 & 154 & Cancer & Breast cancer \\
\hline P21266 & GSTM3_HUMAN Glutathione S-transferase Mu 3 & 26542.2 & 59 & Cancer & Breast cancer \\
\hline P21964 & COMT_HUMAN Catechol O-methyltransferase & 30017.6 & 35 & Cancer & Breast cancer \\
\hline P23921 & RIR1_HUMAN Ribonucleoside-diphosphate reductase large subunit & 90012.5 & 4 & Cancer & Breast cancer \\
\hline P30876 & RPB2_HUMAN DNA-directed RNA polymerase II subunit RPB2 & 133810.7 & 2 & Cancer & \\
\hline P34896 & GLYC_HUMAN Serine hydroxymethyltransferase, cytosolic & 53049.1 & 23 & Cancer & Breast cancer \\
\hline P35520 & CBS_HUMAN Cystathionine beta-synthase & 60548.3 & 9 & Cancer & Breast cancer \\
\hline P61604 & CH10_HUMAN $10 \mathrm{kDa}$ heat shock protein, mitochondrial & 10924.9 & 58 & Cancer & \\
\hline P78417 & GSTO1_HUMAN Glutathione transferase omega-1 & 27548 & 16 & Cancer & Breast cancer \\
\hline Q07973 & CP24A_HUMAN Cytochrome P450 24A1, mitochondrial precursor & 58837.6 & 15 & Cancer & Breast cancer \\
\hline Q13085 & COA1_HUMAN Acetyl-CoA carboxylase 1 & 265382.7 & 1 & Cancer & Breast cancer \\
\hline Q7LG56 & RIR2B_HUMAN Ribonucleoside-diphosphate reductase subunit M2 B & 40710.5 & 5 & Cancer & \\
\hline Q7Z5J4 & RAl1_HUMAN Retinoic acid-induced protein 1 & 203223.9 & 11 & Cancer & \\
\hline Q92820 & GGH_HUMAN Gamma-glutamyl hydrolase precursor & 35941.2 & 17 & Cancer & \\
\hline Q9UBB5 & MBD2_HUMAN Methyl-CpG-binding domain protein 2 & 43228 & 7 & Cancer & Breast cancer \\
\hline Q9UHN1 & DPOG2_HUMAN DNA polymerase subunit gamma-2, mitochondrial precursor & 54876.3 & 7 & Cancer & \\
\hline \multicolumn{6}{|c|}{ Cytoskeleton organization } \\
\hline P07437 & TBB5_HUMAN Tubulin beta chain & 49639 & 230 & Cancer & Breast cancer \\
\hline P62328 & TYB4_HUMAN Thymosin beta-4 & 5049.5 & 15 & Cancer & \\
\hline Q13509 & TBB3_HUMAN Tubulin beta-3 chain & 50400.3 & 127 & Cancer & Breast cancer \\
\hline Q14980 & NUMA1_HUMAN Nuclear mitotic apparatus protein 1 & 238113.2 & 505 & Cancer & Breast cancer \\
\hline Q92747 & ARC1A_HUMAN Actin-related protein 2/3 complex subunit 1A & 41542.7 & 1 & Cancer & \\
\hline \multicolumn{6}{|c|}{ Transport/trafficking } \\
\hline 014908 & GIPC1_HUMAN PDZ domain-containing protein GIPC1 & 36026.7 & 34 & Cancer & Breast cancer \\
\hline O75915 & PRAF3_HUMAN PRA1 family protein 3 & 21600.4 & 19 & Cancer & \\
\hline Q8N1B4 & VPS52_HUMAN Vacuolar protein sorting-associated protein 52 homolog & 82169.8 & 14 & Cancer & \\
\hline
\end{tabular}

pathways, having a role in inhibiting G2/M cell cycle progression under p53 regulation. It modulates the activity of signaling proteins by binding to phospho-Ser/Thr motifs, and was found to be down-regulated in cancer cells. Prohibitin, on the other hand, inhibits DNA synthesis, and is believed to be a negative regulator of cell proliferation. Other players of the DNA replication machinery such as the origin replication complex (ORC) subunits 3,4 and 5 , the mini-chromosome maintenance $(\mathrm{Mcm})$ proteins 2,4 and 7, the replication protein A3 (RPA3 of RFA3) and flap 


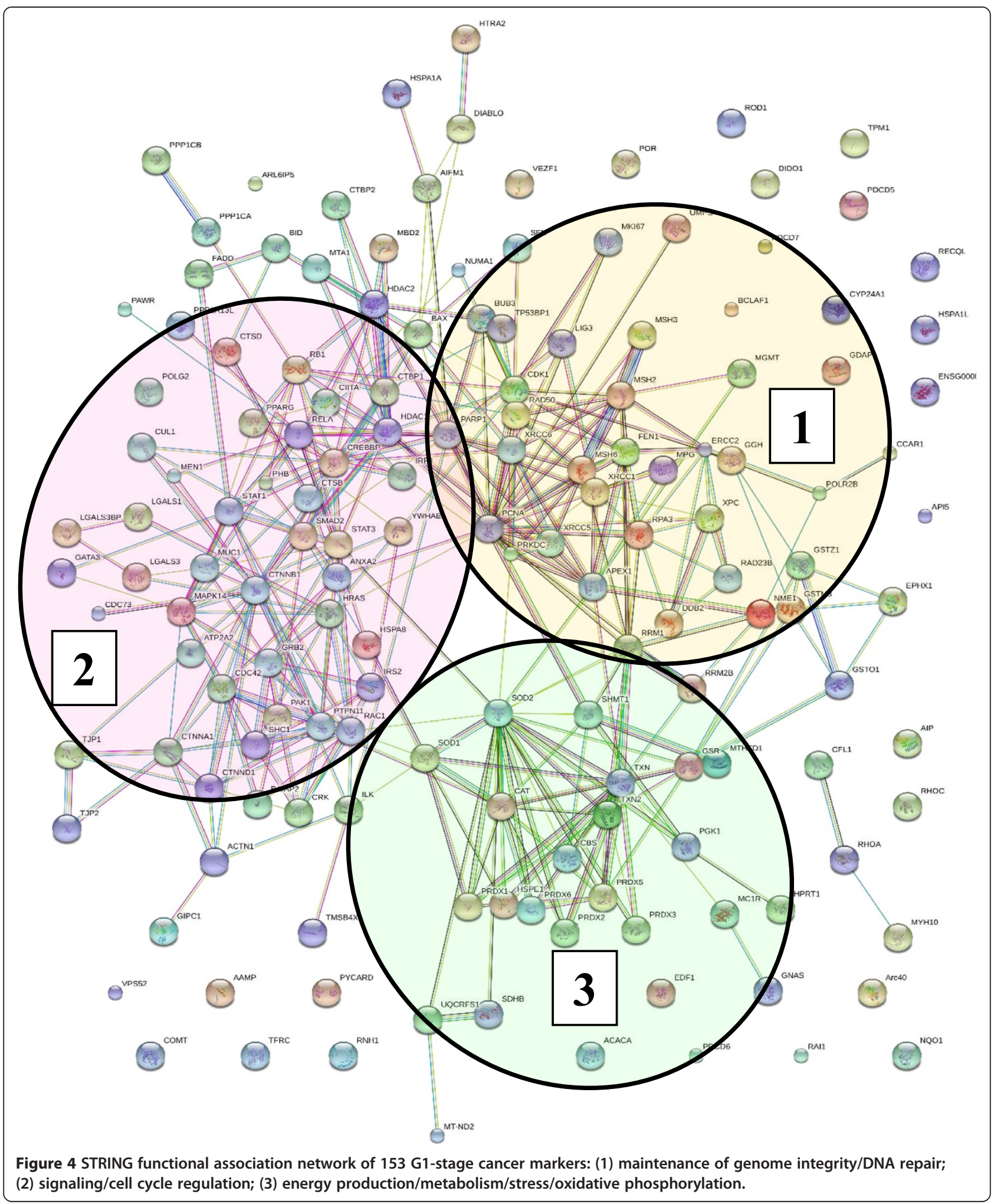

endonuclease 1 (FEN1), as well as key proteins of the proteasomal degradation pathway, were detected. Protein degradation by the $26 \mathrm{~S}$ proteasome pathway is an essential cell cycle regulatory process as it acts as a one-way switch that guarantees correct cell cycle phase transitions [20]. CUL1, a member of the Skp/Cullin/F-box complex (SCF) that controls the levels of CDK inhibitors p21 and p27, was also identified in the data set. Altogether, the data 
suggest that key positive and negative regulators of the cell cycle machinery are identifiable by MS in G1-arrested cells, and that these regulators act in various stages of protein synthesis and degradation, or protein alteration by posttranslational modifications.

\section{Apoptosis}

Apoptosis is a tightly regulated process of cell destruction which cancer cells evade in a variety of ways, and this resistance has been recognized as a hallmark of tumorigenesis $[16,21]$. The extrinsic pathway involves the activation of transmembrane death receptors from the tumor necrosis factor (TNF) superfamily (e.g., FAS and TNF- $\alpha$ ) by death signals/ligands from the cell surface. Ligand binding results in the recruitment of FAS-associated death domain protein FADD which associates with procaspase- 8 causing its activation and entry into the execution phase. The intrinsic pathway is triggered by a variety of stimuli such as growth factor withdrawal, hypoxia and direct DNA damage, among other factors, and acts through the p53 stress sensor. Once phosphorylated by DNA checkpoint proteins (ATM and CHK2), MDM2 mediated ubiquitination and tagging for proteolysis is impeded, and $\mathrm{p} 53$ proceeds to the activation of pro-apoptotic BCL-2 and repression of anti-apoptotic BCL-2 family of proteins. Increased p53 levels also lead to the increase of reactive oxygen species (ROS) that cause mitochondrial damage and release of DIABLO, ARTS and HTRA2 that activate the mitochondrial caspase cascade. The most relevant apoptosis protein markers that were identified included BAX (BCL-2 associated X protein which accelerates programmed cell death by binding to- and antagonizing apoptosis repressor BCL-2), AIFM1 (apoptosis-inducing factor 1), PDCD5/6/7 (with roles in induction and/ or acceleration of apoptosis), DIDO1 (a programmed cell death protein), CCAR1 (a cell cycle and apoptosis regulator), ASC (a caspase-mediated apoptotic factor), HTRA2 (an inhibitor of the activity of inhibitors of apoptosis proteins), BID (BH3-interacting domain death agonist, a pro-apoptotic protein from the BCL-2 family), PAWR (a down-regulator of anti-apoptotic BCL-2), PAK1 (with roles in protection against apoptosis), API5 (an apoptosis inhibitor), and members of the $\mathrm{m}$-TOR signaling pathway proteins with roles in cell survival and evasion of apoptosis [15,21]. Beyond apoptosis, the activities of these proteins have broad ramifications into a multitude of signaling pathways including MAPK, ErbB and p53. BAX and PAK1 represent not only the most interconnected pro- and antiapoptotic markers, but also provide a link between the DNA damage repair, proliferation and signaling protein clusters (see Figure 4).

\section{DNA damage response}

Cells have developed various DNA repair mechanisms to correct for genomic damage caused by replication errors, chemical or environmental factors. The DNA damage repair proteins that were identified in G1 represent a large cluster of interacting proteins in Figure 4, and match the entire range of DNA damage response (DDR) pathways including mismatch repair (MSH), base excision repair (XRCC), nucleotide excision repair (XPC, PNKP, ERCC2), single and double strand break repair (XRCC, TP53BP, RAD50, PARP, PCNA, APEX, LIG3, PRKDC), homologous recombination and nonhomologous endjoining [22]. A manifold of connections among all DNA repair proteins highlights the complex set of mechanisms that were developed by cells to preserve the genome integrity. Proteins with multiple interactions and roles such as PARP1 and PCNA are at the center of the DNA damage response network, and, as noted earlier for the apoptotic proteins, provide the functional link between the DNA damage, proliferation and cell cycle signaling clusters. As PARP1 has been found to be involved in the initiation of ssDNA break repair, and to play a major role in tumor development when dsDNA break repair cannot proceed via BRCA1/ BRCA2 mediated homologus recombination due to various BRCA1/BRCA2 deficiencies, a variety of PARP inhibitors are under development for treating not just cancer, but also stroke and cardiovascular diseases [15].

\section{Angiogenesis}

Angiogenesis is the highly regulated process of new blood vessel formation for the purpose of nutrient and oxygen supply required for cell function and survival [16]. Angiogenesis is a hallmark of cancer, but can be tied to other processes in the cell such as inflammation and wound healing [23]. G1-proteins with roles in angiogenesis included the gene products of MAPK14 (mitogen activated protein kinase), AAMP (angioassociated migratory cell protein), VEZF1 (vascular endothelial zinc finger), RNH1 (RINI) (ribonuclease/ angiogenin inhibitor), STAT3 and p300/CBP (CREBbinding protein) [15]. MAPK14 can be activated by proinflammatory cytokines and through its kinase activity has roles in cell cycle regulation. AAMP has additional roles in cell migration and VEZF1 in transcription regulation and cellular defense response. RNH1 is an angiogenin inhibitor, STAT3 an angiogenesis modulator, while the $\mathrm{p} 300 / \mathrm{CBP}$ proteins act as transcriptional co-activators of the angiogenic factor VEGF (vascular endothelial growth factor) [15,16,23]. Both pro- and anti-angiogenic factors possess various additional biological functions. MAPK14 and STAT3 lie at the heart of multiple signaling pathways that mediate gene expression in response to various stimuli, 
and play central roles in many cellular processes that link angiogenesis to cell growth, proliferation and apoptosis.

\section{Cell adhesion, migration, tissue invasion}

Cell adhesion and migration play important roles in the initiation of tumor invasion and metastasis [24,25]. Several tight junction (TJ) proteins, among which ZO1 and $\mathrm{ZO} 2$, and numerous adherens junction (AJ) proteins, which included $\alpha$ - and $\delta$-catenin, were identified. Tight junctions $(\mathrm{TJ})$ regulate the passage of ions and solutes between cells, while adherens junctions (AJ) participate in the initiation and stabilization of cell-cell contacts. Much broader roles in signal transduction, gene expression, cell cycle modulation and cytoskeleton regulation have been, however, described for these proteins. Transmembrane E-cadherin proteins use their extracellular domain to interact with E-cadherins on adjacent cells, and their intracellular domain to interact with p120-catenin ( $\delta$-catenin/CTND), $\alpha$-catenin (CTNA) and $\beta$-catenin (CTNB). The catenins provide a link to the actin cytoskeleton and signaling pathways. Reduced levels of CTNA have been implicated in epithelial cancers, including breast cancer, and have been associated with increased invasiveness. CTND plays a role in the regulation of cell motility by interaction with RHO GTPases, and it has been implicated in breast cancer progression. On the cell surface, MUC1 (mucin), a membrane-bound Oglycosylated protein, plays additional roles in cell adhesion (the $\alpha$-subunit) and the modulation of intracellular signaling pathways (the $\beta$-subunit) that include ERK, SRC, NF-kappa-B, RAS/MAPK and p53. Changes in the glycosylation pattern or overexpression of this protein have been frequently associated with carcinomas [26].

Cell motility involves cytoskeleton reorganization to extend the cell into the intended direction, as well as sever and create cell adhesions at the trailing and leading edges, respectively [27]. RHO, RAC and CDC42 are well studied cell motility regulators that belong to the RHO subfamily of RAS superfamily of GTPases [6]. Overexpression of these proteins has been linked to progression and metastasis of breast cancer. In addition, CATD, an estrogen regulated aspartyl protease that cleaves substrates such as fibronectin and laminin, has been associated with cell invasion and tumor invasiveness in breast cancer. TYB4 (with roles in actin polymerization), PTN11 (a protein tyrosine phosphatase involved in signaling) and LEG3 (carbohydrate binding) play additional roles in cell adhesion, migration and proliferation, while the expression of the MTA1 protein was correlated with metastatic potential [28]. PAK1, a Ser/Thr p21-activating kinase, is part of a family of proteins that link RHO GTPases to cytoskeleton reorganization and nuclear signaling, serving as targets for the small GTP binding proteins CDC42 and RAC [15]. It is known to regulate cell motility and morphology.

\section{Differentiation}

Cell differentiation, proliferation and cell cycle regulation are processes that work concurrently, but independently, sharing certain key players with individual regulatory function [29]. Differentiation is the process by which unspecialized cells reach a terminal, nonproliferative state by acquiring structural and functional characteristics to perform a specific function [6]. Blocking of differentiation plays an important role in cancer pathogenesis, as poor cell differentiation correlates to more aggressive tumor phenotypes, and viceversa. There is evidence suggesting that the mechanisms that prevent hyperphosphorylation of RB favor differentiation, while mechanisms that promote $\mathrm{RB}$ hyperphosphorylation favor a block in differentiation [6]. Complementing the cell cycle and cell proliferation regulators (RB, MAPK, RAS-related proteins and protein phosphatases), a series of proteins with various roles in differentiation have been identified: GATA3, PPARG, EDF1, NME1 or NDKA, LGALS1 or LEG1, LGALS3, CTNNA1, GDAP1 and ROD1 [15]. GATA3, a transcriptional activator involved in the differentiation of luminal epithelial cells such as MCF-7 and that has been suggested as a breast cancer predictor, displays an inverse correlation to metastasis capability and strong association with estrogen receptors, though it does not appear to be involved in the estradiol signaling pathway [30]. In addition, GATA3 represses adipocyte differentiation by suppressing the peroxisome proliferator-activated receptor $\gamma$ (PPARG). On the other hand, EDF1 (endothelial differentiationrelated factor), a transcriptional activator, stimulates PPARG activities. The NME1, LGALS, CTNNA1 (catenin, alpha1), GDAP1 and ROD1 proteins have roles in inducing or blocking cell differentiation. The overlapping functional relationships between differentiation, invasive properties, angiogenesis and proliferation are clearly observable in Figure 4, and point in the direction of the same signaling proteins, strengthening the relevance of this protein set to determining cell fate.

\section{Oxidative phosphorylation/stress//redox regulation}

Oxidative phosphorylation is an energy-producing metabolic pathway composed of five mitochondrial membranebound multiprotein complexes (I-V) which use the energy generated by electron transfer to synthesize ATP [31,32]. A number of proteins belonging to NADH dehydrogenase, $\mathrm{NADH}$ ubiquinone oxidoreductase and ATP synthase complexes, cytochrome b-c complexes/oxidases, peroxiredoxins and thioredoxins, were identified. Aberrancies in oxidative phosphorylation such as electron leakage leading to oxidative stress, and mutations in these complexes, 
have been reported in cancer. Reactive oxygen species (ROS) formed from leaked electrons play a role in DNA damage and apoptosis, inducing oxidative stress, which in turn can promote angiogenesis and metastasis. Peroxiredoxins and antioxidant enzymes, such as $[\mathrm{Cu}-\mathrm{Zn}]$ superoxide dismutase (SODC or SOD1, and SODM or SOD2) and catalase (CATA) modulate the levels of ROS and are important players in the cellular detoxification processes. While overproduction of ROS induces cell death, moderate levels reportedly can confer resistance to apoptosis and promote cell proliferation. Over expression of SOD1 and CATA has been reported in breast tumors and other types of cancer. APEX1, with roles in cell detoxification and the redox regulation of transcriptional factors, also displays activities in the base excision repair of DNA lesions induced by oxidative and alkylating agents where it functions as an apurinic/apyrimidinic endodeoxyribonuclease [15]. While this group of proteins formed a rather independent cluster of interacting partners in Figure 4 (see cluster 3), the link to the signaling and DNA damage response clusters is clearly evidenced through the superoxide radical capturing SOD1 and SOD2 proteins.

\section{Signaling}

The largest sub-set of cancer markers included 48 proteins with various roles in modulating a broad range of signal transduction events (Table 2). Numerous pathways that promote cell proliferation are activated by the binding of various ligands to cell surface receptor tyrosine kinases (RTKs). SH2-containing proteins such as GRB2, SHC, STAT3 which bind phosphorylated RTKs, were present in the cytoplasmic fractions. GRB2, SHC1 and CRK are known as adaptor proteins because of their specific role as intermediates in protein-protein interactions $[6,16]$. When GRB2 binds a phosphorylated RTK directly or through $\mathrm{SHC1}$, it can initiate a signaling cascade by successive binding of SOS, a guanine nucleotide exchange factor that activates the membrane bound RAS protein by replacing GDP with GTP. Out of four existing mammalian RAS proteins, H-RAS was identified in the cytoplasmic fractions. RAS has a vast list of effector proteins which can activate different signaling pathways, of which the major ones are RAL-GDS, RAC/ RHO, RAF, PI3K and RAL-GEF. The RAF mitogenic pathway has been described as possibly the most relevant to

Table 2 Signaling pathways and associated proteins

\begin{tabular}{|c|c|}
\hline Pathway & Representative proteins \\
\hline MAPK: Proliferation, differentiation, migration & $\begin{array}{l}\text { HRAS/RASH, HSP71, HSP7C, HS71L, NF-kappa- } \beta \text { or TF65/RELA, GNAS, } \\
\text { PAK1, RAC1, SHC1, MAPK14, GRB2, CRK }\end{array}$ \\
\hline $\begin{array}{l}\text { ErbB: Proliferation, differentiation, survival, angiogenesis and adhesion/ } \\
\text { motility/migration/invasion }\end{array}$ & SHC1, PAK1, GRB2, CRK, HRAS \\
\hline Cell cycle: Proliferation & SMAD2, SHC1, 1433s/SFNC/stratifin, HDAC1/2, CREBBP, CUL1, CDC2 \\
\hline DNA damage repair: Maintenance of genome integrity & PARP1, TP53BP, PCNA, PRKDC, DDB2, CREBBP (Table 1) \\
\hline Apoptosis: Cell death & BCLF1, BAX, TF65, IASPP \\
\hline $\begin{array}{l}\text { p53: Cell cycle arrest, apoptosis, senescence, inhibition of angiogenesis/ } \\
\text { metastasis, inhibition of IGF-1/mTOR pathway }\end{array}$ & $\mathrm{BAX}, \mathrm{RIR} 2 \mathrm{~B}, \mathrm{DDB} 2, \mathrm{CDC} 2,1433 \mathrm{~s}$ \\
\hline TGF- $\beta$ : proliferation, apoptosis, differentiation, migration & SMAD2, CREBBP, CUL1, RHOA \\
\hline $\begin{array}{l}\text { NF-kappa-B: Regulation of genes involved in immunity, inflammation, } \\
\text { cell survival }\end{array}$ & NF-kappa- $\beta / T F 65 / R E L A$ \\
\hline Wnt: Cell division, development, adhesion & CTNB1, SMAD2, CTBP2, CREBBP, CUL1, RAC1 \\
\hline $\begin{array}{l}\text { Jak/STAT: Growth, proliferation, development, cell fate, immunity, } \\
\text { cell cycle, apoptosis }\end{array}$ & STAT 3, PTN11, STAT1, GRB2, CREBBP \\
\hline Toll-like: Innate immune responses to pathogenic bacteria & TLR2, IRF3, TF65, MAPK14, STAT1, RAC1 \\
\hline Notch: Proliferative signaling, angiogenesis & HDAC1, CTBP1/2, CREBBP \\
\hline VEGF: Angiogenesis & MAPK14, SHC1, RAC1, HRAS \\
\hline Adhesion/integrin signaling: Cell migration, tissue invasion & $\begin{array}{l}\text { CTNB1, PAK1, GRB2, CRK, HRAS, RAC1, PP1A/B, CDC2, RHOA, ILK, } \\
\text { MUC1, PTN11 }\end{array}$ \\
\hline Ca signaling: Proliferation, apoptosis, metabolism & AT2A2, GNAS1 \\
\hline Insulin signaling: Maintenance of energy metabolism/homeostasis & Q96RG5, TFR1, PTN11, COA1, SHC1, GRB2, CRK, PP1A/B \\
\hline $\begin{array}{l}\text { Chemokine: Immune response, cell growth, differentiation, survival, } \\
\text { migration, apoptosis, regulation of cytoskeleton }\end{array}$ & STAT3, PAK1, TF65, SHC1, GRB2, CRK, STAT1, RHOA, RAC1, HRAS \\
\hline PPAR signaling: Lipid metabolism, adipocyte differentiation & PPARG, ILK \\
\hline Fatty acid biosynthesis & COA1 \\
\hline
\end{tabular}


cancer pathogenesis due to its capability to activate several growth-promoting genes, provide anchorage independence, repress contact inhibition, change cell shape and in general promote proliferation $[6,16]$. Downstream proteins in this pathway include the extracellular regulated protein kinases (ERK) ERK1 and ERK2. Pertinent to the G1/S transition, activation of ERK1 and ERK2 is required up to late G1 for expression of cyclin D1 and successful S entry, though the activity of these proteins is not necessary after the R-point. Further implications of RAS activation involve sustained angiogenesis and evasion of apoptosis. Overall, the deregulation of the SOS-RAS-RAF-MAPK signaling cascade is heavily implicated in acquired growth factor autonomy. In the larger landscape, RTK initiated signaling integrates ErbB, Jak/STAT, integrin, insulin, cell cycle/DNA repair and apoptosis signaling, chemokine signaling integrates Jak/STAT, G-protein and Ca signaling pathways, while Ras, downstream in the cell, further modulates the outcome of TGF- $\beta$, Wnt and NF-kB signaling. The ultimate result is a complex orchestration of crosstalk and positive/negative feed-back loops that control cell cycle progression.

\section{Discussion}

A first important finding of this study reveals that the majority of cancer marker proteins that were compiled in the DAVID disease database from rather random and unrelated studies on cancer, and that were identified in MCF-7 G1 cells, are not isolated players in the development of the disease, but part of multiple regulatory networks that integrate seamlessly with the hallmarks of cancer. A close examination of Figure 4 reveals that the proteins displaying the largest number of interactions (>10-15) also represent the functional links between the three major clusters: SOD1, SOD2 and CAT (from the oxidative stress cluster), PARP1, PCNA and APEX1 (from the DNA damage cluster) and STAT3, RAC1, RELA and ILK (from the signaling cluster). A STRING interaction diagram of this circle of proteins and of a few additional regulatory proteins with $>5$ interactors highlights in detail these central functional relationships (Figure 5). The outcome is conclusive: DNA damage coexists with oxidative phosphorylation and stress, and, in response, multiple signaling pathways work in tandem to determine the fate of the cell. Cancer marker signaling proteins such as STAT3, RAC1, RELA and ILK are involved through their parent pathways essentially in all aspects of signal transduction (Table 2: Jak/STAT, NFkappa- $\beta$, MAPK, Wnt, Toll-like, VEGF, chemokine, integrin), linking a vast array of extracellular stimuli to the intracellular signaling cascades that govern cell proliferation, repair, differentiation, immune response, invasion, metastasis or death. SOD2, SOD1 and CATA are key antioxidant defense enzymes that alleviate the toxic

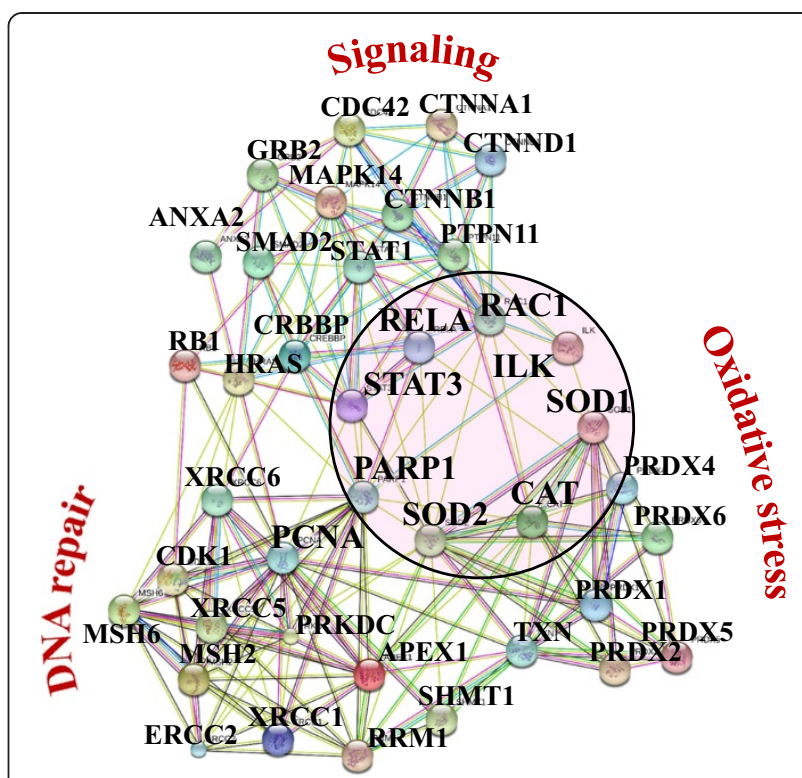

Figure 5 STRING functional association network of central cancer markers that link the DNA repair, signaling and metabolic clusters.

effects of hydrogen peroxide and superoxide anions/radicals produced as a result of various metabolic processes, while PARP1 and PCNA orchestrate DNA replication and damage repair functions to ensure healthy cell proliferation. Alterations in the activity of these genes and their protein products associate inherently, therefore, with the development of cancerous cell states. While members of all three major clusters were represented in both the nuclear and cytoplasmic cellular subfractions, the DNA damage repair components prevailed in the nuclear fraction, while the signaling and cellular detoxification components in the cytoplasm. Based on their functional roles, the nuclear markers and some of the signaling proteins are indicative of a network of drug targets, while the cytoplasmic proteins of a network or putative biomarkers, respectively. In recent years, the value of such a network-based set of markers has been recognized [33,34]. New concepts such as network biomarkers and dynamical network biomarkers have gained popularity, primarily due to the promises brought to improving early diagnostics, sensitivity and specificity, and to behaving more robustly with smaller number of samples [33]. Moreover, the study of network models has suggested that therapies aimed at the inhibition of a number of drug targets, even if small and even if partial, can be much more effective than therapies aimed at the complete inhibition of a single target [34].

The second finding of this study reveals that a common thread of the identified cancer marker clusters is the presence of both agonist and antagonist members within the cluster. The results confirm that entire signaling pathways may have both proliferative and inhibitory 
outcomes. For example, the activation of STAT1 and STAT3 results in opposite effects on tumorigenesis. While STAT3 is considered an oncogene that promotes cell survival/proliferation, motility and immune tolerance, STAT1 is a tumor suppressor through its antiproliferative, pro-apoptotic and angiogenesis-inhibitor activities [23]. The active TGF- $\beta$ signaling pathway has, on the other hand, a role in growth inhibition. Upon activation of the pathway, SMAD2 and SMAD3 undergo phosphorylation in the cytosol followed by binding of either phosphorylated protein to SMAD4, nuclear translocation of the complex, and transcription factor activity. Relevant to cell cycle control, the CDK inhibitors p15 and p21 are the important targets of this pathway [6]. Repression of TGF- $\beta$ signaling is therefore a way in which cancer cells can achieve insensitivity to antigrowth signals. For example, EVI1 and AML1/EVI1 inhibit the transcription factor activity of SMAD3 in the nucleus by direct interaction through a zinc-finger motif. Furthermore, CTBP (C-terminal binding protein), a transcriptional repressor necessary for the inhibition of SMAD3 by EVI1, recruits a histone deacetylase (HDAC) complex which aids in the repression of antigrowth signals [24]. The two CTBP vertebrate homologues (CTBP1 and CTBP2), as well as the histone deacetylases HDAC1 and HDAC2, were identified in the nuclear fractions, suggesting that important participants of acquisition of insensitivity to antigrowth signals are in place in MCF-7 cells. For a complex disease such as cancer, that hosts entirely deregulated but viable signaling pathways, the identification of a novel regulatory network such as suggested by Figures 4 and 5, of its components and of its dynamic behavior, is expected to have a major impact on clarifying the mechanistic details of disease progression.

The third finding of the study reveals that the identified signaling clusters control or modulate not one, but several cancer-related biological processes. Likewise, physiological responses within a cell are elicited not through one, but through multiple signaling pathways. The MAPK and Jak/STAT pathways have ramifications within, virtually, all biological processes that determine the fate of a cell. The delineation of a panel of proteins that modulate cell adhesion/motility/metastasis (CTNB1, MUC1, PAK1, RAC1, RHOA, PTN11, CRK, GRB2, $\mathrm{PP} 1 \mathrm{~A} / \mathrm{B})$, while simultaneously playing central roles in signaling pathways such as MAPK, ErbB, IGF and Jak/ STAT (and, as a result, in all aspects of cell division, proliferation, apoptosis and differentiation, see Tables 1 and 2), is vital to providing insights into the mechanism used by cancer cells to invade adjacent tissues and into the correlation of these processes with events occurring within the cytoplasmic signal-transduction pathways. Simultaneously, the placement of both pro- and antiapoptotic proteins within a broader panorama of cell cycle regulation, survival, proliferation and differentiation proteins is critical to exploring the impact of antiapoptotic factor up-regulation or pro-apoptotic factor down-regulation in cancer. Such perspectives can reveal clues into whether cancer cells have (or not) the ability to activate self-destruction signaling mechanisms, and into the strategies evolved by cancer cells to evade apoptosis and respond to cellular signals that indicate a malfunction of the cell proliferation machinery or the presence of physiological stress. Future protein differential expression studies will be able to validate which networks prevail in the case of particular pathological cancer phenotypes.

\section{Conclusions}

In this work, through proteomic profiling of the G1 stage of MCF-7 cells, and by making use of publically available information and bioinformatics tools, we uncovered a highly interconnected network of nuclear and cytoplasmic cancer markers with regulatory role in biological processes representative of all hallmarks of cancer. Protein interaction analysis and biological characterization of the data revealed that clusters pertaining to cell cycle regulation, signaling, DNA repair, differentiation, angiogenesis and apoptosis, were particularly well represented in the pool of identified proteins. The three major networks formed by these cancer markers, i.e., signaling, maintenance of genome integrity and oxidative stress, are indicative of the different mechanisms that cancer cells utilize to maintain viability in the absence of mitogenic stimulation and to possibly evade the R-restriction point and sustain an aberrant proliferative status. Due to their collective and intertwined roles on the proliferative behavior of cancer cells, the identified markers represent a panel of broad functional relevance to both biomarker and drug discovery research. The combined biomarker/drug-target potential is elevated by the fact that the panel emerged from a list of markers representative of a number of cancerous cell states, compiled rather randomly by on-line bioinformatics tools. The data suggest that proteins with redundant or multiple roles in the control of a cell's fate represent the most informative cancer markers and the most valuable leads for the development of multiplexed biomarker assays and of anti-cancer drugs with increased therapeutic potential.

\section{Additional files}

Additional file 1: Title of data: MCF-7 G1 cell cycle proteins and DAVID functional clustering. Description of data: The table contains proteins identified in the MCF7 G1 stage of the cycle and their DAVID categorization.

Additional file 2: Title of data: List of cancer markers with associated biological and mass spectrometry information. Description of data: The table contains proteins identified in the MCF7 G1 stage of the cycle that 
were associated with the presence of cancer, their GO categorization, disease associations, and Kegg pathways.

\section{Competing interests}

The authors declare no competing interests.

\section{Authors' contributions}

MJT performed the culture and analysis of MCF-7 cells, and drafted a preliminary version of the manuscript. IML conceived the study, coordinated the work, evaluated the overall results, and prepared the final version of the manuscript. Both authors read and approved the final manuscript.

\section{Acknowledgments}

This work was supported by grants from Virginia Tech and $\mathrm{NCl}$

(R21CA126669-01A1) to I. M. Lazar. The content is solely the responsibility of the authors and does not necessarily represent the official views of the National Cancer Institute or the National Institutes of Health. The publication of this article was supported by Virginia Tech's Open Access Subvention Fund.

Received: 17 October 2013 Accepted: 17 September 2014 Published: 24 September 2014

\section{References}

1. Howard A, Pelc S: Synthesis of deoxyribonucleic acid in normal and irradiated cells and its relation to chromosome breakage. Heredity 1953, 6(suppl):261-273.

2. Park M, Lee S: Cell cycle and cancer. J Biochem Mol Biol 2003, 36:60-65.

3. Pardee $A B$ : A restriction point for control of normal animal cell proliferation. Proc Natl Acad Sci U S A 1974, 71:1286-1290.

4. Hartwell LH, Weinert TA: Checkpoints: controls that ensure the order of cell cycle events. Science 1989, 246:629-634

5. Ford HL, Pardee AB: Cancer and the cell cycle. J Cell Biochem 1999, 32-33 (Suppl):166-172.

6. Weinberg RA: The Biology of Cancer. New York: Garland Science, Taylor \& Francis Group, LLC; 2007.

7. Tenga MJ, Lazar IM: A proteomic snapshot of breast cancer cell cycle: The G1/S Transition Point. Proteomics 2013, 13:48-60.

8. Sarvaiya HA, Yoon JH, Lazar IM: Proteome profile of the MCF-7 cancer cell line: a mass spectrometric evaluation. Rapid Commun Mass Spectrom 2006, 20:3039-3055.

9. Yang X, Lazar IM: MRM screening/biomarker discovery: a library of human cancer-specific peptides. BMC Cancer 2009, 9:96.

10. Gasteiger E, Gattiker A, Hoogland C, Ivanyi I, Appel RD, Bairoch A: ExPASy: The proteomics server for in-depth protein knowledge and analysis. Nucleic Acids Res 2003, 31:3784-3788.

11. Zeeberg BR, Feng W, Wang G, Wang MD, Fojo AT, Sunshine M, Narasimhan S, Kane DW, Reinhold WC, Lababidi S, Bussey KJ, Riss J, Barrett JC, Weinstein JN: GoMiner: a resource for biological interpretation of genomic and proteomic data. Genome Biol 2003, 4:R28.

12. Huang DW, Sherman BT, Lempicki RA: Systematic and integrative analysis of large gene lists using DAVID bioinformatics resources. Nat Protoc 2009, 4:44-57.

13. Dennis G Jr, Sherman BT, Hosack DA, Yang J, Gao W, Lane HC, Lempicki RA: DAVID: Database for Annotation, Visualization, and Integrated Discovery. Genome Biol 2003, 4:P3.

14. Jensen LJ, Kuhn M, Stark M, Chaffron S, Creevey C, Muller J, Doerks T, Julien $P$, Roth $A$, Simonovic M, Bork $P$, von Mering C: STRING 8-a global view on proteins and their functional interactions in 630 organisms. Nucleic Acids Res 2009, 37:D412-D416

15. Safran M, Dalah I, Alexander J, Rosen N, Iny Stein T, Shmoish M, Nativ N, Bahir I, Doniger T, Krug H, Sirota-Madi A, Olender T, Golan Y, Stelzer G, Harel A, Lancet D: GeneCards Version 3: the human gene integrator. Database 2010, 2010. 1-16. Article ID baq020, doi:10.1093/database/baq020.

16. Hanahan D, Weinberg RA: The hallmarks of cancer: the next generation. Cell 2011, 144:646-674.

17. Kanehisa M, Goto S: KEGG: Kyoto Encyclopedia of Genes and Genomes. Nucleic Acids Res 2000, 28:27-30.
18. Hochegger H, Dejsuphong D, Sonoda E, Saberi A, Rajendra F, Kirk J, Hunt T, Takeda S: An essential role for Cdk1 in S phase control is revealed via chemical genetics in vertebrate cells. J Cell Bio/ 2007, 178:257-268.

19. Polanski M, Anderson NL: A list of candidate cancer biomarkers for targeted proteomics. Biomark Insights 2006, 1:1-48.

20. Diehl JA, Ponugoti B: Ubiquitin-dependent proteolysis in G1/S phase control and its relationship with tumor susceptibility. Genes Cancer 2010 1:717-724.

21. Elmore S: Apoptosis: A review of programmed cell death. Toxicol Pathol 2007, 35:495-516

22. Ciccia A, Elledge SJ: The DNA damage response: making it safe to play with knives. Mol Cell 2010, 40:179-204.

23. Grivennikov SI, Greten FR, Karin M: Immunity, inflammation and cancer. Cell 2010, 140:883-899.

24. McSherry EA, Donatello S, Hopkins AM, McDonnell S: Molecular basis of invasion in breast cancer. Cell Mol Life Sci 2007, 64:3201-3218.

25. Gumbiner BM: Cell adhesion: the molecular basis of tissue architecture and morphogenesis. Cell 1996, 84:345-357.

26. Varki A, Kannagi R, Toole BP: Glycosylation changes in cancer, chapter 44 In Essentials of Glycobiology. 2nd edition. Edited by Varki A, Cummings RD, Esko JD, Freeze HH, Stanley P, Bertozzi CR, Hart GW, Etzler ME. New York: Cold Spring Harbor Laboratory Press; 2009.

27. Braga V: The crossroads between cell-cell adhesion and motility. Nat Cell Biol 2000, 2(10):E182-E184.

28. Futamura M, Nishimori $H$, Shiratsuchi T, Saji S, Nakamura Y, Tokino T: Molecular cloning, mapping and characterization of a novel human gene, MTA1-L1, showing homology to a metastasis-associated gene, MTA1. J Hum Genet 1999, 44:52-56.

29. Brown G, Hughes PJ, Michell RH: Cell differentiation and proliferationsimultaneous but independent? Exp Cell Res 2003, 291:282-288.

30. Chou J, Provot S, Werb Z: GATA3 in development and cancer differentiation: cells GATA have it! J Cell Physiol 2010, 222:42-49.

31. Lemarie A, Grimm S: Mitochondrial respiratory chain complexes: apoptosis sensors mutated in cancer. Oncogene 2011, 30:3985-4003.

32. Brown NS, Bicknell R: Hypoxia and oxidative stress in breast cancer. Oxidative stress: its effects on the growth, metastatic potential and response to therapy of breast cancer. Breast Cancer Res 2001, 3:323-327.

33. Liu R, Wang X, Aihara K, Chen L: Early Diagnosis of Complex Diseases by Molecular Biomarkers, Network Biomarkers, and Dynamical Network Biomarkers. Med Res Rev 2013, in press.

34. Csermely P, Ágoston V, Pongor S: The efficiency of multi-target drugs: the network approach might help drug design. Trends Pharmacol Sci 2005 26:178-182.

doi:10.1186/1471-2407-14-710

Cite this article as: Tenga and Lazar: Proteomic study reveals a

functional network of cancer markers in the G1-Stage of the breast cancer cell cycle. BMC Cancer 2014 14:710.

\section{Submit your next manuscript to BioMed Central and take full advantage of:}

- Convenient online submission

- Thorough peer review

- No space constraints or color figure charges

- Immediate publication on acceptance

- Inclusion in PubMed, CAS, Scopus and Google Scholar

- Research which is freely available for redistribution 